\title{
Utilization Fuzzy Logic in Agriculture Sprinkler System
}

Nirsandh Ganesan ${ }^{1}$, Vishwathi kumaresan ${ }^{2}$, Ramya Devi $U^{3}$

${ }^{1,2}$ Research Engineer, Research and Development board, KEDS GROUPS, Coimbatore, Tamilnadu. India.

${ }^{3}$ Research scholar, Depart of Mthematics, Poompuhar College, Melaiyur, Nagapattinam, Tamilnadu.

India.

nirshanthkodai@gmail.com ${ }^{1}$,vishwathikumaresan@gmail.com ${ }^{2}$

\begin{abstract}
This water management system enhances irrigated agriculture usage. We implemented an open loop fuzzy logic based scheme in this system. Inside the field, the inputs to the fuzzy logic system are modified from a pressure transducer, a temperature controller, and a flux sensor. The light and the water pump are the device's inputs. The simulation is performed with the aid of MATLAB and Simscape tools. The controller's control parameters and rule-based schemes are illustrated in the following sections of this study.
\end{abstract}

Keywords: Fuzzy logic, sprinkler, IF-THEN Rules, Rainfall prediction

\section{Introduction}

Agriculture is a big origin of income in many nations and has a significant impact on economies. In most cropping schemes, a systematic approach to water conservation is a top priority. Inside the case of inadequate rain or even during dry periods, agriculture is incredibly hard, possibly requiring irrigation for better yield and nutrient preservation. Water is necessary to life to continue. Plants require a specific quantity of water; too many or too few liquid may harm plant growth. Food scarcity is a recurring issue that cannot be addressed solely by rain-fed agricultural activity. Food poverty persists in the majority of countries, not to mention the ongoing water crisis. Whenever the production of food rises, further irrigation could be used to help alleviate acute shortages of food. Power supplies were draining, and groundwater consumers should be subject to certain restrictions. This suggests that the agricultural sector's water consumption could be limited to $33 \%$ by 2025 . This necessarily requires more orderly water use in irritability. Irrigation qualities which were calculated at $27 \%$ in 1990 could increase to $54 \%$ by 2025 in order to reduce water use in irrigation. [1]. Irrigation controllers are classified into two major categories:
Controller for an open loop: There is no error input from the managed object for this kind of controller. The user determines the start and finish times, as well as the stop cycles and watering hours. The criteria that have been set are [2]:

- The length of the agriculture session

- The frequency at which the irrigation cycle should be repeated.

- Closed system controls for measuring an amount of money needed in agriculture: These are all the controls which provide input combined to input forward into the monitored entity and then have the information to quantify the amount of money needed for agriculture. As just a consequence, even as weather changes, the volume of moisture in use for agriculture increases. [2].

- Irrigation is a form of irrigation. Closed loop controller decisions are founded on [3]:

- Paying attention to the state variables

- Distinguishing between state variables and expected variables.

- Identifying which steps are required to alter the device condition.

- Take the necessary actions. An occurrence close PID controller program reacts automatically to climatic and ecological shifts, due to high irrigation efficiency. [3]. 
www.rspsciencehub.com

The system's input parameters are as follows:

Temperature

- Relative humidity

- Sunshine Illumination

- Irradiation by the Sun

- The Wind Speed Output parameter is as follows:

- Water pump speed.

\section{Related Process}

"A Live Data Design of an Arduino modem Ejector Refrigeration Control Scheme utilizing irrigation Systems Research methods," by VeenaDivya with AyushAkhouri, could have the capabilities of maintaining uniform environmental conditions [4].Its Android os that comprises OS, software, and core programs has been used on gadgets. The Android Operating system provides the tools and APIs you'll have to get starting creating Android applications using the Java language. This programme uses the GPRS functionality of cell phones as an irrigation control system solution. This scheme targeted a smaller variety of agricultural land and was not commercially viable.

Purnima and S.R.N Reddy conducted a study titled "Development of Remote Monitoring and Control System with Automatic Irrigation System Using GSM-Bluetooth," and we suggested supplying groundwater to ground when food is grown artificial [5]. The primary sources for irrigation water are outdated arm motors, canal water, and runoff. There are many disadvantages to this process, both under irrigation and over cultivation, which results in leaching and nutrient depletion.

To address this problem, the fuzzy logic approach and over half of the amount was protected using a mobile phone network system. As a result, the increased yield became the cause.

The paper 'Fuzzy Logic-Based Image Classification"by I. Nedeljkovic aids in the arrangement of SPOT images using fuzzy logic. It demonstrates the use of spectral details for specific agricultural ground cover groups. [6] Formalized paraphrase to be more specific, Matlab introduces factors for inputs (image channels) and output (land classes). The membership feature was determined on the basis of a monitored recognition conducted using PCI Image Works. Those fuzzy inference system classification principles then are obtained utilizing Mat lab's Fuzzy Logic Toolkit, and that they are validated or supported through designed to simulate identification procedures in random sample areas. Finally, spot image recognition was worked out. Fuzzy logic has demonstrated that it could be successfully used during classification tasks via experiments conducted, leading to higher necessary consequence.

The article "Recognition of Weeds with Image Processing and Their Use with Fuzzy Logic for Precision Farming" [7] describes the technique created for processing digital images to create a weed diagram. According to the researchers, the fuzzy logic solution can ensure that image data is converted into sprayer commands, and the available fuzzy logic controller can only operate one sprayer. This study shows that the greenness approach can automatically locate weeds and apply herbicides regulated by fuzzy logic to achieve successful weed control. T. A. Izzuddin et al. used fuzzy logic to introduce irrigation that optimizes water use for agriculture. The inference engine control system was used to apply an open loop fuzzy logic control system. Matlab and Simulink were used for simulation. The findings showed that fuzzy logic system was correctly modeled, and also that the mamdani design of fuzzy logic control system maximizes seed water consumption [8].

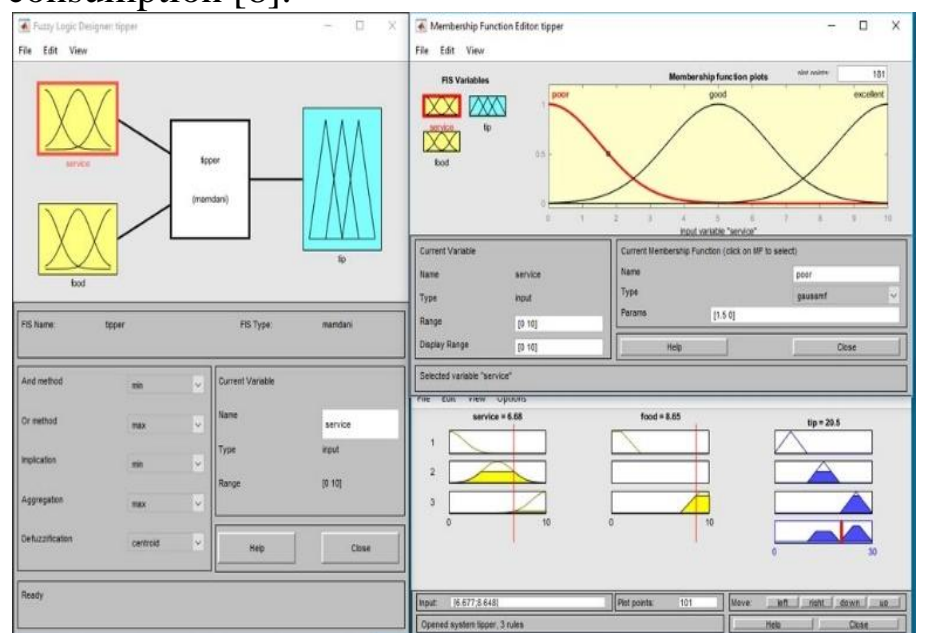

Fig.1 Fuzzy logic interface

Our suggested smart irrigation scheme would use an open loop system and a near loop fuzzy logic system that is based mostly on Mamdani and Sugeno control fuzzy reasoning schemes. It'll be implemented in Matlab as well as the results compared and see which fuzzy inference system is by far the most effective. A Fuzzy Logic Controller is represented as just a block diagram. 


\section{www.rspsciencehub.com}

The Fuzzy Inference System's most important component is indeed the fuzzy reasoning unit, which would be composed of a knowledge base and national database. The fuzzy stage converts or incorporates outputs into true value that are then calculated by knowledge base. The output values would be browned up and during defuzzification phase. Sugeno output is the crisp number obtained by multiplying each input value by either a fixed rate but instead combining those two outcomes. [7$10]$.

\section{Proposed Model}

\subsection{Fuzzy logic control system}

Fig.1 depicts the structure of a fuzzy logic control scheme. Similar to [9], the system is composed of four blocks: Fuzzification, inference engine, ifthen law, and defuzzification. The system's inputs would be analogue values. The Fuzzification, on the other hand, would allocate the crisp inputs into fuzzy sets. Along with the fuzzified sets, the ifThen logic is fed into the inference engine. The fuzzified inputs are then assigned to various sets based on the membership functions. The controller's performance is the defuzzified shape in crisp form.

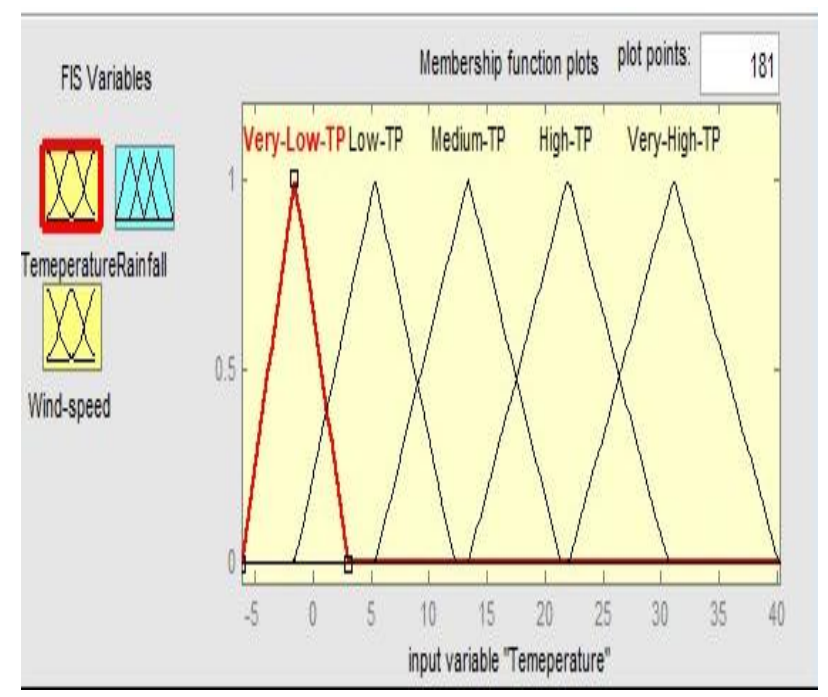

Fig.2 Plot: Temperature

In fig.2 FIS variables and process denotes the temperature function plots. This figure has denoted the temperature function plot.

Crisp details Crisp inputs are indeed the five signals loaded into the machine (temperature, humidity, sunlight, solar irradiation, and wind speed). Unpredictable nature a sharper quality is fuzzy through such a mechanism. To put it differently, it reflects the conversion of a clear
Volume 03 Issue 04 April 2021

attribute it into fuzzy package. It procedure's main goal is to turn numerical output into fuzzy sets. Because it improves the program's robustness and efficiency, Gaussian membership can be included.

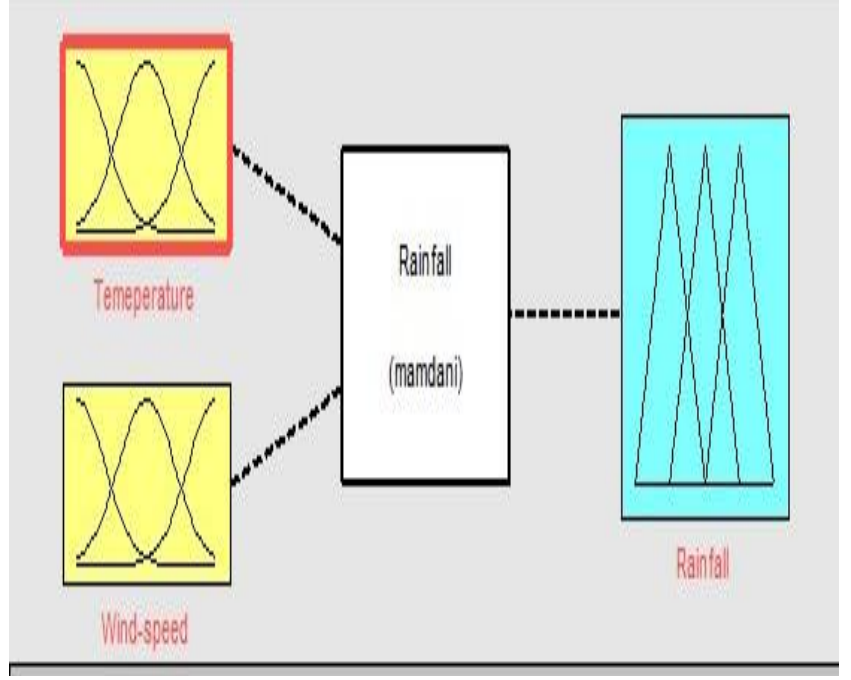

Fig 3: Temperature and wind speed Fuzzification process

In fig 3 FIS variables and process denoted the two function plots (Temperature, Wind speed).Both the values are processed to find the most précised data to predict the rain fall condition.

Table.1: Threshold level

\begin{tabular}{|l|l|l|}
\hline $\begin{array}{l}\text { Serial } \\
\text { number }\end{array}$ & Temperature & Threshold $^{\mathbf{0}}$ \\
\hline 1 & Low & {$\left[\begin{array}{ll}0 & 14\end{array}\right]$} \\
\hline 2 & Medium & {$\left[\begin{array}{lll}0 & 14 & 30\end{array}\right]$} \\
\hline 3 & High & {$\left[\begin{array}{lll}14 & 30\end{array}\right]$} \\
\hline
\end{tabular}

Table. 1 is shows the acquired threshold values for temperature. Here the threshold value is 0 to 14degree Celsius or below 14 it will marked as Low. If the value is above 14 or 14-30 degree Celsius it will marked as medium temperature. If the threshold value is 14-30 degree Celsius or above 30 degree Celsius it will marked as high temperature. Here the temperature plays a vital role in prediction system. Generally low temperature is the best sign of low humidity level.

\subsection{Inference Engine and Rule Base}

It is made up of a series of rules that reflect the problem's knowledge base and reasoning structure. To generate a fuzzy value, the fuzzy engine will add rules from the rule base. 
Rule Editor: Rainfall

File Edit View Options

1. If (Temeperature is Very-Low-TP) and (Wind-speed is Very-Low-WS) then (Rainfall is Very-Low-RF) (1)
2. If (Temeperature is Low-TP) and (Wind-speed is Low-WS) then (Rainfall is Very-Low-RF) (1)
3. If (Temeperature is Very-Low-TP) and (Wind-speed is Very-High-WS) then (Rainfall is Medium-RF) (1)
4. If (Temeperature is Very-High-TP) and (Wind-speed is Very-High-WS) then (Rainfall is High-RF) (1)
5. If (Temeperature is Very-Low-TP) and (Wind-speed is Medium-WS) then (Rainfall is Low-RF) (1)
6. If (Temeperature is Very-Low-TP) and (Wind-speed is High-WS) then (Rainfall is Low-RF) (1)
7. If (Temeperature is Very-Low-TP) and (Wind-speed is Medium-WS) then (Rainfall is Low-RF) (1)
L

Fig.4: IF rules Temperature

Here IF rulesused to control the water pump condition and it will directly manipulate the water irrigation system in agriculture land.

The rule base contains laws that have been modelled using information and practice. For this query, there are five input variables, each with three distinct linguistic regions.

\subsection{If-then rules}

The if-then rules are created in Matlab "Rules" section. These are shown in Fig. 5 \& 6 .

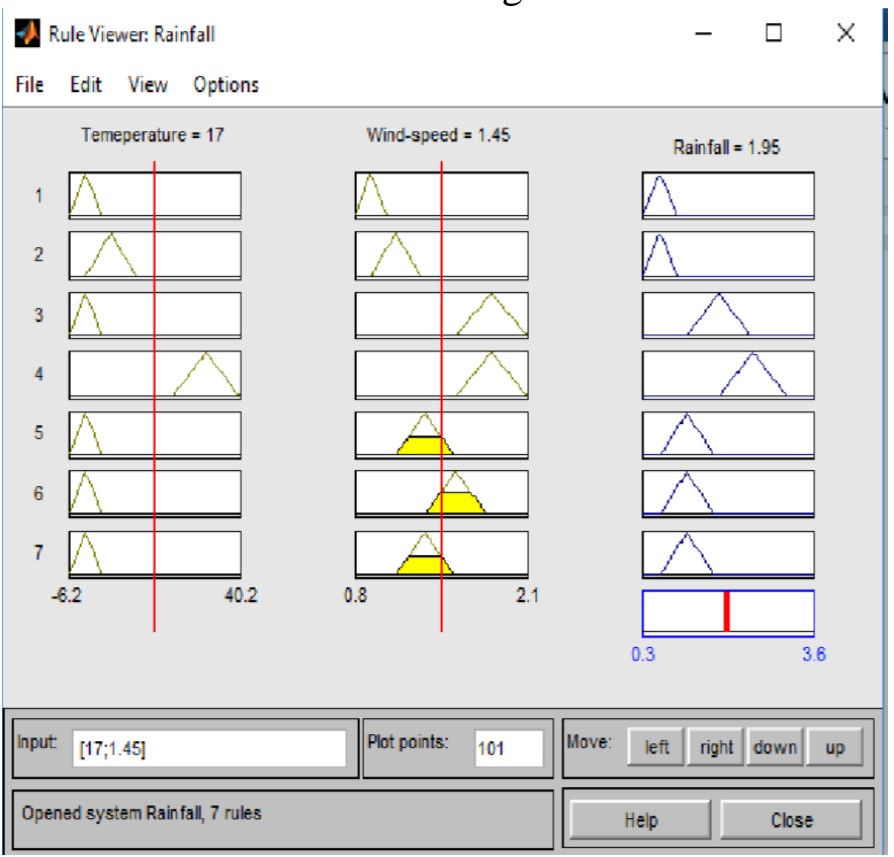

Fig.5: Rainfall plots

The Fig. 5 denoted that the system has calculate the temperature value and wind speed to predict the upcoming weather condition finally compared with both FIS variables the final output showed by the system. In this process we got the average temperature 17 Degree Celsius and wind speed is 1.45 final output of rainfall condition is 1.95 .

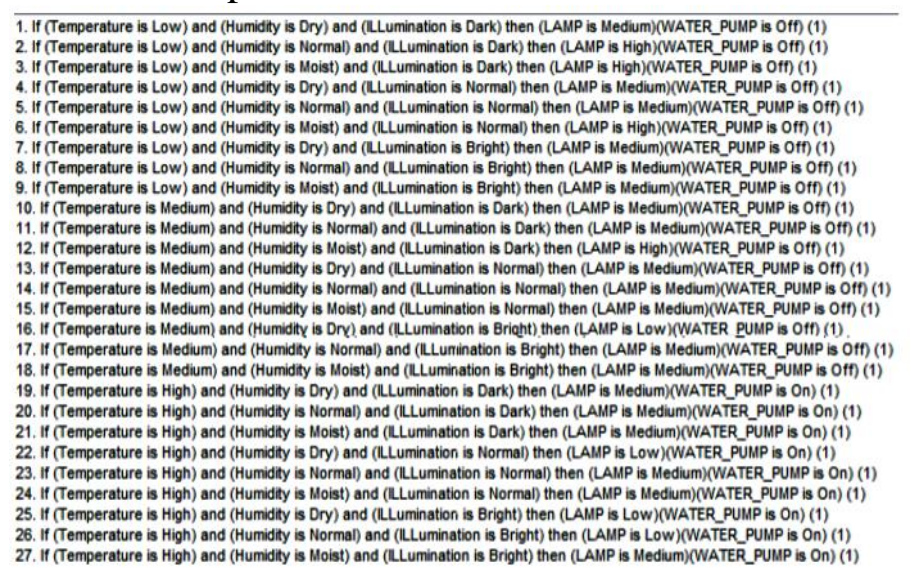

\section{Fig.6: IF -THEN Rules}

If indeed the (air temp) and (wind direction) are very small (output is very low).

- If (this same humidity is exceptionally low) and (the wind speed is exceptionally low) (output is very low).

- Unless (the weather is incredibly cold) and (this same velocity is regular) (output is low).

- When (the humidity is relatively low) and (the wind speed is incredibly high) (output is medium).

- When (the weather is relatively low) and (the wind direction is incredibly high) (output is very low).

\section{Discussion}

To start, a suggested method would increase cropwat efficiency through having an irrigation system more efficient and reliable, hence why inputs factors include temperature; humidity levels, daylight lighting, irradiance, and wind direction are being used to run the cooling system. The system then will calculate that irrigation threshold based on five input variables, but table 2 will describe that threshold in details.

Table.2 Labels and parameters of fuzzy variables

\begin{tabular}{|c|c|c|}
\hline $\begin{array}{c}\text { Fuzzy } \\
\text { variables }\end{array}$ & Parameters & Linguistic Labels \\
\hline 1 & Temperature, TP & Very high TP, High TP, Medium TP, Low TP, Very Low TP \\
\hline 2 & Wind speed, WS & Very high WS, High WS, Medium WS, Low WS, Very low WS \\
\hline 3 & Rainfall, RF & Very high RF, High RF, Medium RF, Low RF, Very low RF \\
\hline
\end{tabular}


Table.3 Input parameters of fuzzy variables

\begin{tabular}{|c|c|c|c|c|c|c|c|}
\hline $\begin{array}{c}\text { I/O } \\
\text { parameters }\end{array}$ & $\begin{array}{c}\text { Temper } \\
\text { ature }\end{array}$ & $\begin{array}{c}\text { Relative } \\
\text { humidity }\end{array}$ & $\begin{array}{c}\text { Sunshine } \\
\text { Illumination }\end{array}$ & $\begin{array}{c}\text { Solar } \\
\text { irradiation }\end{array}$ & $\begin{array}{c}\text { Wind } \\
\text { speed }\end{array}$ & $\begin{array}{c}\text { Water } \\
\text { Pump } \\
\text { (Mamdani) }\end{array}$ & $\begin{array}{c}\text { Water } \\
\text { Pump } \\
\text { (Sugeno) }\end{array}$ \\
\hline Best & 28 & 75 & & 9.5 & 28 & 328 & 399 \\
\hline Average & 15 & 40 & 5 & 15 & 1.5 & 61.2 & 0.108 \\
\hline Worst & 2 & 5 & 0.5 & 2 & 0.2 & 55.4 & $5.08 * 10-14$ \\
\hline
\end{tabular}

Table.3 has mention the FIS variables, these parameters are Temperature, Relative humidity, Sunlight, solar radiation, Wind speed. Above parameter are factors to manipulate the irrigation pipes control system to maintain the proper humidity level. Here the system categorized as three levels Best, Average, Worst. Compared with our proposed method to sugeno method, our proposed method is getting more accurate value to predict the parameter to manipulate the irrigation system.

\section{Conclusion}

There's been a plan for cost-effective irrigated areas. It article was an intelligent irrigation device which took into consideration input variables and class labels to make it more efficient and successful. The main aim of this study would be to create a fuzzy logic algorithm joystick for agriculture production software updates. A series of membership values was added to Mat lab's fuzzy logic control system .The quantities $29.8,62,215$ were added to the input parameters of temperature, humid, and illuminated, and demonstrates 344 and 722, separately, crisp output of the fuzzy logic upon on Lamp and Water Pump.

\section{References}

[1].Esener, I.I., Yukksel, T., \&Kurban, M., (2015). "Short term load forecasting without metrological data using AI-based structures", Turk. J. Electr. Eng. Comput. Sci., 370-380.

[2].Singla, M.K., Nijhawan, P., Oberoi, A.S., \& Singh, P., (2019). "Application of Levenberg Marquardt Algorithm for Short Term Load Forecasting: A theoretical investigation", Pertanika J. Sci. \&Technol, 27(3), 1227-1245.

[3].Kandari, A.A., Soliman, S., \&Hawary, E.I., (2004). "Fuzzy short-term electric load forecasting”, Int J Electr Power Energy Syst 26(2), 111- 122.
[4].Feinberg, E.A., \&Genethliou, D., (2005). "Load forecasting. Applied mathematics for restructured electric power systems", Chap 12. Springer, New York, 269-285.

[5].Fan, S., \& Hyndman, R.J., (2012). "Short-term load forecasting based on a semi-parametric additive model", Power Syst IEEE Trans, 27(1), 134-141.

[6].Singla, M.K, (2018). "Load Forecasting Using Artificial Neural Network" (Doctoral dissertation).

[7].Singla, M.K., \& Gupta, J., (2018). "Load Forecasting Using Back Propagation Algorithm", International Journal of Engineering and Techniques, 4, 169-175.

[8].Singla, M. K., \& Hans, S., (2018). "Load Forecasting using Fuzzy Logic Tool Box", Global Research and Development Journal for Engineering, 38, 12-19.

[9].Singla, M.K., Gupta, J., \&Nijhawan, P., (2019). "Comparative Study on Backpropagation and Levenberg Marquardt Algorithm on Short Term Load Forecasting", International Journal of Advanced Trends in Computer Science and Engineering, 8(2), 194202.

[10].Singla, M.K., Gupta, J., Nijhawan, P., \&Oberoi, A.S., (2019). "Electrical Load Forecasting Using Machine Learning", International Journal of Advanced Trends in Computer Science and Engineering, 8(3), 615619.

[11].Esener, I.I., Yukksel, T., \&Kurban, M., (2015). "Short term load forecasting without metrological data using AI-based structures", Turk. J. Electr. Eng. Comput. Sci., 370-380. 
[12].Singla, M.K., Nijhawan, P., Oberoi, A.S., \& Singh, P., (2019). "Application of Levenberg Marquardt Algorithm for Short Term Load Forecasting: A theoretical investigation", Pertanika J. Sci. \&Technol, 27(3), 1227-1245.

[13].Kandari, A.A., Soliman, S., \&Hawary, E.I., (2004). "Fuzzy short-term electric load forecasting", Int J Electr Power Energy Syst 26(2), 111- 122.

[14].Singla, M.K., \& Gupta, J., (2018). "Load Forecasting Using Back Propagation Algorithm", International Journal of Engineering and Techniques, 4, 169-175.

[15].Singla, M.K., Gupta, J., \&Nijhawan, P., (2019). "Comparative Study on Backpropagation and Levenberg Marquardt Algorithm on Short Term Load Forecasting", International Journal of Advanced Trends in Computer Science and Engineering, 8(2), 194202. 